\title{
Les mesures de débits effectuées en France à l'aide de traceurs radioactifs par la méthode d'intégration
}

\author{
Discharge measurements carried out in France \\ by the integration method with radioactive tracers
}

PAR R. HOURS,

INGÉNIEUR, ADJOINT AU CHEF DE LA SECTION DES APPLICATIONS DES RADTOÉLÉNAENTS, COMMISSARIAT a E'ÉNERGIE ATOMIQUe de SAGLaY

J. GUISERIX,

G. GRANDCLEMENT,

INGÉNIEURS A LA SECTION DES APPLICATIONS DES RADIOÉLÉMENTS CENTRE D'ÉTUdes NuClÉatres de GRenoble

H. ANDRÉ,

INGENIEUR A L'ÉLECTRICITÉ DE FRANCE, DIYISION TECHNIQUE GÉNÉBALE, GRENOBLE

\author{
R. WOLF ET R. PÉREZ, \\ INGÉNIEURS A L'ÉLECTRIGITÉ DE FRANGE \\ CENTRE DE RECHERCHES ET D'ESSAIS DE CHATOU
}

\begin{abstract}
Les études entreprises en France, depuis denx années, sur les mesures de débits d'eau, à l'aide de radioéléments par la méthode d'intégration, ont porté sur la recherche des conditions d'application et sur le choix du matériel nécessaire à ces mesures.

Après le rappel du principe de la méthode, des conditions fondamentales d'emploi et des différents traceurs, le processus des mesures est donné sous forme détaillée ainsi que la description du matériel utilisé. Le mémoire se termine par l'exposé des principaux essais réalisés.
\end{abstract}

French research on this subject during the past two years has been concerned with the determination of conditions under which the method can be applied and the type equipment required.

After reviewing the principle of the method, the basic conditions governing its use and the varions tracers employed, the anlhors give a detailed description of the actual measurement procedure and the equipment. The article concludes with an exposé of the principal tests to which it has been subjecled. 


\section{1. - CADRE DE CETTE ÉTUdE. CHOIX DE LA MÉTHODE}

Les études entreprises en France depuis deux années sur la mesure de débits d'eau à l'aide de traceurs radioactifs avaient pour objet d'évaluer les possibilités de la méthode décrite par D.E. Hull, en 1958, à la conférence de Genève sur les Applications Pacifiques de l'Energie Atomique, connue en France sous le nom de méthode de dilution - procédé par intégration, et qualihé dans les pays anglo-saxons de "gulp method», «total count method ou de sudden injection method $\gg[1]$.

En même temps, était entreprise une investigation de la «méthode d'Allen *, connue dans les pays anglo-saxons sous le nom impropre de «peak timing method» ou de \& pulse dilution methods; nous ne faisons que la mentionner.

Ces études ont été menées en collaboration étroite par le Commissariat à l'Energie Atomique et deux organes de l'Electricité de France : le Centre de Recherches et d'Essais de Chatou (C.R.E.C.) et la Division Technique Générale (D.T.G.). Elles ont porté sur la recherche des conditions d'application de la méthode et sur le choix du matériel nécessaire aux mesures.

Les méthodes de dilutions comparées avec utilisation des sels classiques en 1963, tel que le bichromate de sodium (méthode classique - régime permanent et méthode d'intégration) se- raient lourdes et chères. Chères car, à l'encontre des traceurs radioactifs, le prix du traceur croît linéairement avec le débit à mesurer; lourdes, car le poids de sel (hichromate de sodium) à injecter varie de 0,2 à $2 \mathrm{~kg} / \mathrm{m}^{3} / \mathrm{s}$. Un des caractères recherchés est, enfin, la légèreté du procédé, légèreté qui doit favoriser la multiplicité des mesures.

Avant d'aborder des essais en vraie grandeur, nous avons fait choix, pour mettre au point les différentes parties technologiques de la méthode, qui constituent, à notre sens, une phase essentielle de cette étude, de terrains simples où nous disposions de moyens classiques de référence.

Ainsi, dans la voie des mesures de débits de grands canaux, nous avons effectué un certain nombre de jaugeages de débits en conduites forcées d'usines hydroélectriques, tandis que, dans le but de mesurer ultérieurement des débits de fleuves, nous avons ròdé la méthode radioactive sur les bords de rivières dont les débits étaient de l'ordre de quelques mètres cubes par seconde.

La méthode d'intégration a été choisie pour la simplicité du matériel d'injection, en particulier dans le cas des écoulements de grande largeur. De plus, elle nécessite de deux à trois fois moins de traceur que la méthode de dilution classique.

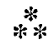

\section{2. - LES TRACEURS EN COMPÉTITION}

Nous avons jugé utile de rappeler les principaux avantages et inconvénients des différents traceurs possibles. Assurément, et ce sera l'idée qui se dégagera de cette analyse, seule la rhodamine $B$ semble être actuellement en compétition avec la radioactivité pour la mesure de forts débits.

\subsection{Le bichromate de sodium.}

Les méthodes de dilutions comparées de bichromate de sodium ont fait en France, ou elles ont vu le jour, l'objet d'un très grand nombre d'essais, et sont aujourd'hui utilisées systématiquement pour le jaugeage en rivière. Elles présentent donc un premier avantage, celui d'être bien connues. Le temps ayant contribué à les parfaire, elles sont actuellement les plus légères en matériel pour la mesure de débits de rivières inférieurs à $20-50 \mathrm{~m}^{3} / \mathrm{s}$. La très grande solubilité du bichromate $(600 \mathrm{~g} / \mathrm{l})$ peut être considérée comme un avantage de ce sel relativement à la rhodamine $B$, ainsi que nous le montrerons plus bas.

La concentration nécessaire pour son dosage est de l'ordre de $2.10^{-7} \mathrm{~kg} / \mathrm{I}$ pour des eaux assez claires, ce qui est souvent le cas des eaux en conduites forcées, et de 5 à $15 \cdot 10^{-7} \mathrm{~kg} / 1$ en général pour les mesures de débits en rivières. La dilution étant de l'ordre de $10^{\circ}$ entre les points d'injection et de prélèvement en rivière, ceci explique la masse importante de $2 \mathrm{~kg} / \mathrm{m}^{3} / \mathrm{s}$ déjà mentionnée pour un fleuve. Il en résulte une masse et un prix prohibitifs pour les mesures de forts débits.

Le risque de réduction du chrome par les ber- 
ges ou les particules en suspension présente un inconvénient, car il est nécessaire de réoxyder le chrome à sa valence la plus élevée et ceci allonge le dépouillement des mesures.

Ajoutons enfin, comme inconvénient notable, mais mineur, que le chrome nécessite pour son dosage un réactif dont les propriétés évoluent avec le temps, et enfin qu'il est nécessaire de conserver les échantillons dans le noir, car ils subissent une transformation sous l'action du soleil.

Nous rappellerons enfin que le bichromate de sodium est maintenant préféré à d'autres sels, toujours dosés par voie chimique, comme le sulfate de manganèse, le chlorure de sodium, principalement parce qu'il conduit au volume le plus faible de solution injectée, et également parce qu'on ne le rencontre pas à l'état naturel dans les rivières, et que son dosage colorimétrique ne fait appel qu'à un personnel peu initié.

\subsection{La rhodamine $B$.}

De grands espoirs sont nés avec les premiers essais de la rhodamine B dont l'étude se poursuit actuellement dans de nombreux laboratoires.

Son extraction des solutions, avec par exemple de l'alcool isoamylique, permet de diminuer de deux à trois ordres de grandeur, par rapport au bichromate de sodium, la concentration minimale nécessaire à son dosage $\left(10^{-9} \mathrm{~kg} / 1\right)$ et, de là, la masse de produit à injecter.

Cet avantage est cependant moins marqué que semblent le montrer ces chiffres car la rhodamine $B$ est moins soluble dans l'eau que le bichromate, et il en résulte que le gain en masse de solution à injecter n'est que de 20, facteur très intéressant cependant.

Si par ailleurs, la rhodamine $B$ montre une très grande stabilité chimique, il apparaîtrait qu'elle soit assez absorbée par les matières en suspension, ce qui serait un grave défaut pour son utilisation dans les fleuves en général très chargés.

Notons enfin qu'un spectrophotomètre de précision, matériel assez délicat, est nécessaire pour son dosage.

\subsection{Chlorure de sodium et mesure de résis- tivité.}

La connaissance de la concentration de CINa par une mesure de résistivité, in situ et en continu, représente un double intérêt de cette méthode. Cependant deux reproches principaux peuvent lui ètre faits. En premier lieu, le niveau souvent assez élevé de la conductivité naturelle des eaux conduit à l'injection de masses de sel trop importantes. En second lieu, les variations dans le temps et dans l'espace de cette résistivité affectent fréquemment ces mesures. De plus, la nécessité de connaîlre la température de l'eau alourdit quelque peu la méthode : nous savons en effet que la résistivité de l'eau varie environ de $-2,5 \% / \mathrm{d}^{\circ} \mathrm{C}$.

\subsection{Les traceurs radioactifs.}

A notre connaissance c'est à $D$. E. Hull que revient le mérite d'avoir le premier présenté la méthode d'intégration avec des traceurs radioactifs. La mesure in situ et en continu, est, dans ce cas, également possible à l'aide d'une sonde immergée et cela peut être considéré comme un avantage pour certaines études, par exemple du temps d'arrivée et du temps de passage de la vague de traceur.

La radioactivité apporte d'autres avantages. Le volume de la solution à injecter est incomparablement plus faible qu'avec les traccurs salins classiques, et le tritium sous forme d'eau tritiée laisse espérer une solution lorsque les traceurs salins actifs ou inactifs subissent une adsorption préjudiciable à la mesure. De surcroît, le prix des traceurs radioactifs, qui croît moins vite que le débit à mesurer, est un avantage intéressant à souligner.

Mais l'on peut reprocher aux traceurs salins radioactifs utilisés en général en hydrologie, le poids des enceintes de protection destinées à leur transport, et par ailleurs, le phénomène de décroissance d'activité pour les traceurs à courte période, phénomène qui conduit à préparer et à transporter une activité bien supérieure à celle qui est effectivement utilisée.

Le tritium ne présente pas cet inconvénient et l'eau tritiée paraît donc la mieux appropriée aux mesures de débits importants. Ccpendant, une très grande prudence doit être recommandée, prudence qui doit proscrire toutes les mesures faisant appel au tritium, s'il peut être montré qu'une contamimation durable des nappes en aval du point d'injection est possible. Cette contamination pourrait handicaper on interdire dans ce milieu d'éventuelles études avec le tritium naturel ou d'origine thermonucléaire.

La mesure du tritium impose le prélèvement d'échantillons et leur comptage en laboratoire avec un appareil assez délicat et cher.

Une dernière particularité importante de la méthode d'intégration avec des traceurs radioactils, à inscrire au chapitre des inconvénients, est la difficulté de connaître le rapport entre l'activité injectée et une activité aliçuote destinée à l'étalonnage du système de détection. La détermination de ce rapport, qui varie de $10^{5}$ à $10^{6}$, fait appel en général à un dispositif spécialisé, important, en particulier dans le cas d'émetteurs de rayonnement gamma dur. 


\section{3. - PRINCIPE DE LA MÉTHODE CONDITIONS FONDAMENTALES}

\subsection{Principe de la méthode.}

Une certaine quantité de traceur radioactif d'activité A est injectée dans la rivière, le canal ou la conduite, selon une loi quelconque en fonction du temps.

A une distance suffisamment grande en aval, un comptage de la vague d'activité est réalisé à l'aide d'une sonde placée en regard - soit de l'écoulement (méthode directe) - soit d'une déviation de l'écoulement (méthode d'échantillonnage continu). Une troisième méthode consiste à effectuer le comptage d'un échantillon moyen, prélevé à débit constant, pendant le passage de la vague d'activité (méthode de prélèvement ou méthode de l'échantillon moyen).

Nous considérerons en premier lieu la méthode directe.

Soit une sonde $\mathrm{S}$ et un volume démentaire $\mathbf{J}$ dans l'eau du volume sensible de cette sonde.

Pendant un temps T supérieur ou égal au passage de la vague d'activité, passe en J une activité :

$$
d A_{j}=\int_{1,} c d Q d t
$$

Le débit élémentaire $d Q$ élant supposé constant (régime permanent en moyenne) nous pouvons écrire :

$$
\frac{d \mathrm{~A}_{j}}{d \mathrm{Q}}=\int_{\mathrm{l}^{2}} c d t
$$

Nous supposerons l'existence d'une relation linéaire entre le taux de comptage $n$ enregistré à l'aide de la sonde $\mathrm{S}$, et dù à l'activité contenue dans le volume $\mathrm{J}$, et la concentration $\mathrm{C}$ dans ce volume ćlémentaire :

$$
\mathrm{C}=\frac{n}{\mathrm{~F}_{j}}
$$

F, représente le taux de comptage pour une concentration unitaire en $\mathrm{J}$.

Nous écrirons alors:

$$
\begin{aligned}
\frac{d \mathrm{~A}_{j}}{d \mathrm{Q}}=\int \frac{n}{\mathrm{~F}_{j}} d t= & \frac{\mathrm{N}_{i}}{\mathrm{~F}_{j}} \\
& \text { en posant } \mathrm{N}_{j}=\int_{\mathrm{T}} n d t,
\end{aligned}
$$

ou encore :

$$
\mathrm{N}_{i}=\mathrm{F}_{i} \frac{d \mathrm{~A}_{i}}{d \mathrm{Q}}
$$

Si $d A_{j} / d Q$ est constant dans tout le volume qui est «vu» par le détecteur, c'est-à-dire $\int c d t=$ constante (et ce sera la condition de «bon mélange $\gg)$, le nombre total de coups $\mathbf{N}$, enregistré pendant le passage de la vague active est :

$$
\mathrm{N}=\int \mathrm{N}_{i} d \omega=\frac{d \mathrm{~A}_{j}}{d \mathrm{Q}} \int \mathrm{F}_{i} d \omega=\frac{d \mathrm{~A}_{j}}{d \mathrm{Q}} \mathrm{F}
$$

En posant :

$$
F=\int_{\omega} F_{j} d \omega
$$

et comme:

$$
\frac{d \mathrm{~A}_{i}}{d \mathrm{Q}}=\text { Cite, } \frac{d \mathrm{~A}_{j}}{d \mathrm{Q}}=\frac{\mathrm{A}}{\mathrm{Q}}
$$

s'il y a conservation de l'activité, et :

$$
Q=F \frac{A}{N}
$$

où l'on a :

$\mathrm{N}=$ nombre total de coups enregistrés;

$A=$ activité injectée;

$\mathrm{Q}=$ débit à mesurer;

$F=$ taux de comptage donné par une activité unitaire dans tout le volume intéressé.

Ce coefficient peut donc être déterminé par un étalonnage dans une géométrie strictement identique à celle dans laquelle est effectuée la mesure.

Une activité $\mathrm{A}_{e}$ est alors introduile dans une enceinte de volume $\mathrm{V}$ et l'on obtient dans ces conditions un taux de comptage $f_{e}$.

On obtient alors :

$$
\mathrm{F}=\frac{f_{e}}{\mathrm{~A}_{e}} \mathrm{~V}
$$

et :

$$
\mathrm{Q}=\frac{\mathrm{A}}{\mathrm{A}_{e}} \frac{f_{e}}{\mathrm{~N}} \mathrm{~V}
$$

avec :

$A=$ activité injectée durant l'essai;

$\mathrm{A}_{e}=$ activité injectée lors de l'étalonnage;

$\mathrm{F}_{e}=$ taux de comptage durant l'étalonnage;

$N=$ nombre de coups enregistrés pendant l'essai;

$\mathrm{V}=$ volume de la cuve d'étalonnage.

Cette identité entre la géométrie de mesure et la géométrie d'étalonnage pose, dans sa réalisa- 
tion, un problème important et souvent difficile.

Il apparaît que, pour une rivière ou un fleuve, la meilleure solution est de placer la sonde dans des conditions de «volume infini », c'est-à-dire dans le volume au-delà duquel la solution active contribue, par exemple, à moins de $1 \%$ du signal.

Ces conditions ne sont pas toujours aisées à respecter, comme nous le montrerons plus loin. De plus, dans le cas de conduites de forts diamètres, on ne dispose paśs souvent d'éléments de conduites pour l'opération d'étalonnage, opération qui serait d'ailleurs fort lourde de mise en œuvre.

Certains préfèrent donc la méthode d'échantillonnage continu à la méthode directe. Une partie du courant principal est dérivée, en général à l'aide d'une pompe, dans une cuve comprenant la sonde de mesure. L'étalonnage est effectué dans cette même cuve et par conséquent dans une géométrie identique.

Les relations établies plus haut sont valables pour cette méthode et la condition de bon mélange est toujours $\int_{\mathrm{T}} c d t$ dans la section de prélèvement.

Nous avons préféré pour nos essais la méthode de prélèvement, exposée en détail plus bas, dans laquelle on effectue un prélèvement à débit constant $q$ dans une cuve où est ensuite mesurée la concentration moyenne du traceur.

On fait l'hypothèse que la relation de bon mélange $\int_{\mathrm{T}} c d t=$ Cte dans la section de prélèvement est respectée; ceci entraîne :

$$
\frac{\mathrm{Q}}{q}=\frac{\mathrm{A}}{a}
$$

avec :

$$
\begin{aligned}
& \mathrm{Q}=\text { débit à mesurer; } \\
& q=\text { débit de prélèvement; } \\
& \mathrm{A}=\text { activité injectée; } \\
& a=\text { activité prélevée. }
\end{aligned}
$$

Au cours de l'étalonnage une activité $\mathrm{A}_{e}$ donne une fréquence de comptage $f_{\epsilon}$. Si, de plus, le prélèvement présente un taux de comptage $f$, on peut écrire :

$$
\frac{a}{\mathrm{~A}_{e}}=\frac{f}{f_{e}}
$$

et il vient :

$$
\mathrm{Q}=\frac{\mathrm{A}}{\mathrm{A}_{c}} \frac{f_{e}}{f} \cdot q
$$

\subsection{Conditions fondamentales.}

\subsubsection{Condition DE BON MÉLANGe.}

Les relations (1) et (2) ont été établies en faisant l'hypothèse qu'en tous les points du volume de mesure ou de la section de prélèvement, l'expression $\int_{\mathrm{T}} c d t$ est constante. En conséquence, les masses de traceur qui traversent les différents tubes de courant doivent se répartir comme les débits.

\subsubsection{Conservation de la matrère OU DE L'ACTI- VITÉ.}

Pour établir les équations (1) et (2), nous avons utilisé la relation $d \mathrm{~A} / d \mathrm{Q}=\mathrm{A} / \mathrm{Q}$, que nous avons écrite en faisant l'hypothèse qu'il y avait conservation de l'activité; il est donc nécessaire que toute l'activité injectée passe dans la section de mesure ou de prélèvement.

\subsection{Remarques sur les conditions d'applica- tions de la méthode.}

\subsubsection{CAS D'UN AFFLUENT ENTRE LA SECTION D'IN- JECTION ET LA SECTTON DE MESURE.}

Dans la méthode de dilution classique (steady dilution method), il ne doit pas exister d'affluent entre la section d'injection et la section de mesure. Il n'en est pas de même pour la méthode d'intégration. Il sulfit seulement que la distance entre le confluent et la zone de mesure soit suffisante pour que la condition de bon mélange soit respectée.

\subsubsection{Cas D'UnE dérivation DU CoURant PRIN- CIPAL.}

Les démonstrations des méthodes de prélèvement faites plus haut montrent que la mesure peut ètre réalisée sur une partie dérivéc du courant principal. Nous rappellerons seulement que l'expression $\int_{\mathrm{T}} c d t$ doit être constante dans la section de la dérivation. La « distance de bon mélange» concerne ici la distance entre la section d'injection et la section de dérivation.

\section{4. - PROCESSUS DE LA MESURE}

Dans ce chapitre, nous décrivons les différents processus de mesure de débits correspondant à nos propres essais sur les conduites forcées et des rivières de faibles débits. Nous ne ferons que de courtes allusions aux autres méthodes d'intégrations radioactives, et des suggestions pour l'application de la méthode à la mesure de forts débits. 


\subsection{Prise de partie aliquote de l'activité principale.}

Nous insisterons dans ce paragraphe sur l'étalonnage des sondes de détection, question qui n'est, à notre sens, pas assez souvent mise en relief.

Dans les équations (1) et (2) apparait le rapport $\mathrm{A} / \mathrm{A}_{\mathrm{e}}$ entre l'activité injectée durant la mesure et l'activité injectée pour l'étalonnage.

Si l'on désire connaitre le débit avec une précision de quelques pour cent, ce rapport doit être connu avec une précision de quelques pour mille. Il est important de noter par ailleurs qu'il est difficile de comparer deux activités qui diffèrent de 4 à 6 ordres de grandeur.

Si l'utilisateur peut faire appel à un laboratoire de mesures radiométriques spécialisé, le problème est simplifié pour lui, car il reçoit des activités étalonnées. Il nè doit alors veiller qu'aux variations des efficacités de ses sondes entre les dates d'étalonnage et de la mesure, le mieux étant, bien entendu, que ces dates soient les plus voisines possibles.

$\mathrm{Ne}$ disposant pas, de laboratoire spécialisé dans ce type de mesure, la solution que nous avons adoptée consiste à préparer par dilution volumétrique deux solutions dont les concentrations sont connues. Ces opérations doivent être conduites à distance et derrière un mur de protection. Nous utilisons aujourd'hui une enceinte spécialisée dans laquelle le remplissage, le rincage et le séchage de pipettes étalonnées sont réalisés à distance. Cette enceinte se trouve dans une pièce thermostatée.

Des précautions sont prises pour éviter l'adsorption du traceur sur la verrerie en la «saturant $\gg$ au préalable avec une solution concentrée de sel entraîneur.

Les deux échantillons d'aclivité sont enfin recueillis et transportés dans des bouteilles en verre, fermées avec une bille de verre scellée par une résine. Ces bouteilles, qui seront cassées dans le milieu de l'utilisation, ont été également saturées et contiennent les mêmes quantités de sel actif, d'entraîneur et d'eau.

\subsection{Détermination de la distance de bon mélange, de la date et de la durée de prélèvement.}

Il est difficile de prévoir la distance minimale entre la section d'injection et la section de mesure nécessaire pour obtenir un «bon mélange». On peut admettre que cette distance est la même que celle qui est requise pour réaliser un bon mélange dans la méthode d'injection à débit constant. Dans celte méthode, en effet, la condition de bon mélange est que la concentration du traceur soit la même dans tous les points de la section de mesure: $\mathrm{C}=$ constante. Or, on démontre que l'hypothèse $\mathrm{C}=$ constante entraine $\int_{\mathrm{F}} c d t=$ constante. Inversement, on montre que si la distance est telle que $\int_{\mathrm{T}} c d t=$ constante, on a $\mathrm{C}=$ constante pour une injection continue.

Il est alors possible d'estimer théoriquement la distance que nous appellerons distance de bon mélange.

\subsubsection{Distance De bon MÉlange.}

\subsubsection{Mesures de canaux:}

On suppose que le traceur est injecté à l'aide d'une rampe placée au-dessus de la surface de l'eau et perpendiculaile à la direction de l'écoulement afin de faciliter le mélange dans le sens transversal. La longueur de bon mélange $L$ (telle que les concentrations ne diffèrent pas de plus de $1 \%$ pour une injection à débit constant) est donnée par la formule du C.R.E.C. [2] :

$$
\mathrm{L}=9,5 \mathrm{nh}
$$

où $h$ est le tirant d'eau;

$n$ est le rapport de la vitesse moyenne à la vitesse de frottement. (Cette «vitesse» de froltement est définie par : $\mathbf{U}_{*}=\sqrt{g} \overline{\mathrm{R}} i$ avec

$g=$ accélération de la pesanteur,

$\mathrm{R}=$ rayon hydraulicue;

$i=$ perte d'énergie par unité de poids et par unité de longueur du canal.

On peut prendre pour $n$ :

$$
n=0,32 \mathrm{KR}^{1 / 6}
$$

où $\mathbf{K}$ est le coefficient de Strickler;

$\mathrm{R}$ est le rayon hydraulique.

Unités du système S.I. (mètre, seconde).

On a pu obtenir une bonne vérification expérimentale de cette formule théorique.

\subsubsection{Mesures en rivières et fleuves:}

Dans ce cas, et pour une injection ponctuelle faite dans l'axe de l'ćcoulement, la formule établie par Rimmar [3] permet d'estimer la distance minimale de bon mélange; cette formule ne semble pas encore avoir été systématiquement contrôlée, et il apparaît qu'elle ne devrait donner qu'un ordre de grandeur

$$
\dot{\mathrm{L}}=0,13 \mathrm{~N} \cdot \frac{b^{2}}{h}
$$

où $b$ est la largeur moyenne de la section mouillée dans la section de mesure (en mètres);

$h$ est la profondeur moyenne de l'eau dans cette section (en mètres); 


$$
\begin{aligned}
& \mathrm{N} \text { est un coefficient égal à } \mathrm{C} \frac{(0,7 \mathrm{C}+6)}{g}, \\
& \mathrm{C}=\text { coefficient de Chézy }(15<\mathrm{C}<50) \text {, } \\
& g=\begin{array}{l}
\text { accélération de la pesanteur } \\
\mathrm{m} / \mathrm{s}-2) .
\end{array}
\end{aligned}
$$

\subsubsection{Mesures en conduite forcée:}

La condition pour la distance de bon mélange, établie par MM. Dumas, Lièvre et Bonnin de l'Université de Grenoble est :

$$
\mathrm{L}=75 d
$$

avec $d=$ diamètre de la conduite; et pour une injection dans l'axe de la conduite, ou quatre points d'injection dans une section.

\subsubsection{DATE DU DÉButT DE PRÉLÈvEMENT.}

Si la connaissance de la date du début de comptage dans le cas d'une sonde immergée ne pose pas de problème, elle demeure une question importante dans le cas d'un prélèvement. En effet, afin de ne diluer qu'au minimum l'activité recueillie, le prélèvement devrait débuter à l'arrivée des premières molécules d'activité au point de prélèvement et cesser lorsque passent les dernières molécules actives. Dans la pratique, de larges tolérances sur ces dates doivent être prises.

\subsubsection{Mesures en canal et fleuve :}

Nous ne connaissons pas encore de méthode satisfaisante permettant de déterminer la date de début de prélèvement dans le cas d'un fleuve. Pour les canaux, les caractéristiques hydrauliques de l'usine hydroélectrique permettent d'apprécier le débit, donc la vitesse moyenne. On sait par ailleurs que les premières molécules de traceur arrivent dans un certain temps, de $10 \%$ inférieur à celui que permet de prédire la vitesse moyenne.

\subsubsection{Mesures en conduite forcée :}

Le débit peut être estimé connaissant les conditions hydrauliques; on peut en déduire une vitesse moyenne de la vague d'activité et la date d'arrivée de l'activité selon la règle que nous venons d'indiquer.

\subsubsection{Mesures de petites rivières :}

II semble que la meilleure méthode soit d'effectuer un essai préliminaire avec de la fluorescéine. On peut en effet facilement apprécier à l'œil l'arrivée de la tête de la vague colorée à la zone de mesure.

Une méthode plus lourde, mais plus sûre pour un observateur non expérimenté, utilise l'information délivrée par une sonde nucléaire immergée en amont du point de prélèvement.

\subsubsection{TEMPS DE PRÉlèveMENT.}

4.2.3.1. Mesures en canal, et en conduite forcée :

On montre, pour une injection instantanée, que $99,9 \%$ de la masse du traceur injecté passent en un temps $\mathrm{T}$ égal à (formule du C.R.E.C.) :

$$
\mathrm{T}=9,3\left(\frac{m h x}{n \bar{u}^{2}}\right)^{1 / 2}
$$

où :

$$
\begin{aligned}
m= & \text { coefficient adimensionnel de la disper- } \\
& \text { sion longitudinale, égal à } 7,3 \text { pour les } \\
& \text { canaux et } 10,1 \text { pour les conduites; } \\
h= & \text { tirant d'eau; } \\
x= & \text { distance de la section de mesure à la sec- } \\
& \text { tion d'injection; } \\
\bar{u}= & \text { vitesse moyenne; } \\
n= & \text { rapport de la vitesse moyenne à la vi- } \\
& \text { tesse de frottement. }
\end{aligned}
$$

on peut prendre $0,32 \mathrm{KR}^{1 / 6}=n$

$\mathrm{K}=$ coefficient de Strickler;

$\mathrm{R}=$ rayon hydraulique.

Unités du système S.I. (mètre, seconde)

\subsubsection{Mesures du fleuve:}

Nous n'avons pas encore établi de raisónnement systématique qui conduirait à la détermination du temps de passage de la vague d'activité. Nous pensons, à ce sujet, que cet intervalle de temps, de même aussi que la distance de bon mélange, pourra être déterminé à la suite d'une analyse mathématique basée sur la connaissance expérimentale des coeflicients de diffusion turbulente. La mesure effective du débit serait donc précédée d'une mesure des coefficients de diffusion turbulente, ce qui alourdirait la méthode. Mais rappelons que la mesure d'un débit de fleuves demeurera toujours une opération assez peu fréquente et onéreuse, pour laquelle on peut donc admettre une certaine lourdeur.

\subsubsection{Mesures de riviere:}

Dans la méthode que nous exploitons, le temps de prélèvement doit être fixé avant l'opération elle-même, car nous faisons usage d'orifices calibrés qui déterminent le temps nécessaire pour remplir un volume donné. I a méthode la plus simple semble être alors de procéder à une injection préliminaire de fluorescéine et d'apprécier le temps de passage de la vague colorée à la section de mesure. On a coutume alors de doubler ce temps pour évaluer le temps de passage effectif, car l'œil apprécie mal les faibles concentrations de ce traceur coloré au début et surtout à la fin de la vague.

\subsection{Injection du traceur.}

L'injection du traceur ne pose pas de problèmes difficiles pour les faibles débits. Les difficultés apparaissent pour les débits importants. 


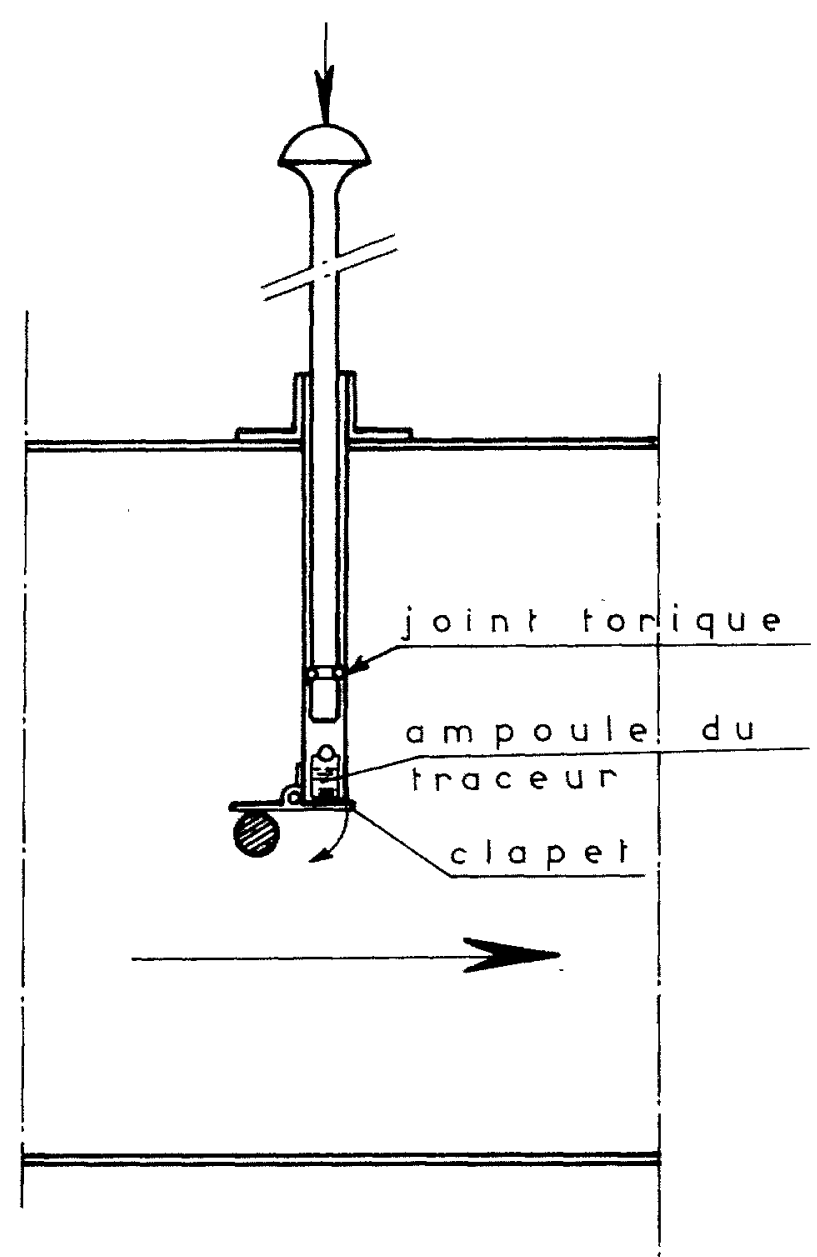

FIG. 1

Dispositif d'injection en conduite.

\subsubsection{CAS DEs condutes forcÉes.}

Le dispositif que nous utilisons permet de casser l'ampoule de verre contenant le traceur dans la conduite elle-même. La figure 1 représente un schéma de ce dispositif, qui est monté en général dans la chambre des vannes en amont de la conduite. La pression est de l'ordre de quelques kilogrammes par centimètre carré. La canne étant enlevée, l'ampoule est introduite dans le conduit. Ce conduit est fermé à sa partie inférieure par une soupape qui est maintenue contre son siège par l'effet du contrepoids et de la pression. La canne est ensuite enfoncée brusquement, elle casse l'ampoule en fin de course, et réalise l'ouverture de la soupape.

\subsubsection{Cas d'Une RiviĖre.}

Une canne de $4 \mathrm{~m}$ de longueur permet d'extraire l'ampoule de son enceinte de protection en plomb. Son extrémité est ensuite immergée el l'ampoule est brisée à l'aide d'un système du type arbalète et commandé à distance (fig. 2).

\subsubsection{Cas des fleuves fet des canaux.}

Nous ne disposons pas encore de système d'injection dans le cas de forts débits. Une injection ponctuelle serait assurément la plus simple, mais il est souhaitable, en vue de diminuer la distance de bon mélange, d'injecter l'activité à la surface d'une section transversale. Le temps d'injection peut ètre théoriquement quelconque, mais l'on a l'avantage à le minimiser, car il détermine en partie le temps de passage de la vague d'activité dans la section de mesure.

L'injection transversale pose un problème important de protection dans le cas d'un traceur émetteur de rayonnement gamma : avec le tritium, une injection à débit constant depuis une embarcation traversant la rivière d'une rive à l'autre pourrait être une solution.

\subsection{Détection et comptage du traceur.}

\subsubsection{CoMptage AVEC UNE SONDE IMMERgÉE.}

La méthode la plus simple de comptage de l'activité est d'immer"ger la sonde de détection. Nous avons vu plus haut, au sujet de l'étalonnage des sondes, qu'il doit y avoir identité absolue entre la géométrie de la mesure et celle de

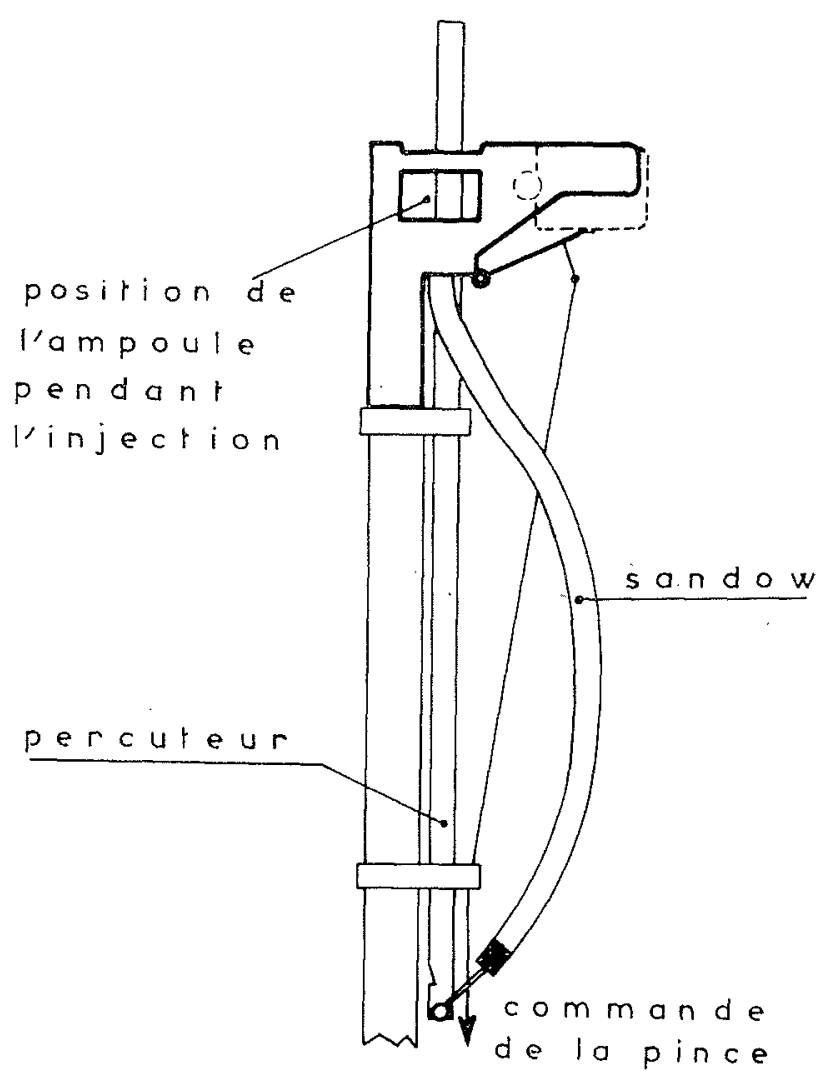

Fra. 2

Détail de la canne d'injection en rivière. 
l'étalonnage. On peut pour cela placer la sonde dans des conditions de «milieu infini », c'est-àdire telles que toutes les parties du volume sensible de la sonde se trouvent dans l'eau. Les rayons infinis sont respectivement de 50, 70, $100 \mathrm{~cm}$ pour $\mathrm{I}^{131}, \mathrm{Br}^{82}$ et $\mathrm{Na}^{24}$; il faut donc par exemple avec le brome 82 que la sonde se trouve au moins a $70 \mathrm{~cm}$ du fond ou de la surface d'une rivière. Il est prudent d'augmenter ces valeurs de $20 \%$.

Sur la figure 3 , nous avons représenté trois dispositifs que nous avons essayés et qui ont été conçus pour faciliter la mise en place de la sonde en milieu infini. En $(\alpha)$, figure une sonde suspendue à un flotteur, à une distance de celuici supérieure au rayon infini. Ce dispositif est d'une mise en œuvre très malaisée. En $(b)$, nous avons représenté un autre dispositif dans lequel la sonde est liée à deux flotteurs par un système rigide. Cette solution est viable, mais, comme dans la précédente, on ne possède aucune indication sur la distance de la sonde au fond de la rivière. En (c), figure un dispositif un peu plus lourd, mais qui une fois immergé donne l'assurance que le détecteur se trouve dans les conditions de volume infini; la sonde se trouve sur l'axe d'un treillis constitué de barreaux d'aluminium représentant un prisme droit dont la base est un triangle équilatéral. Son usage est à recommander pour les rivières à faible tirant
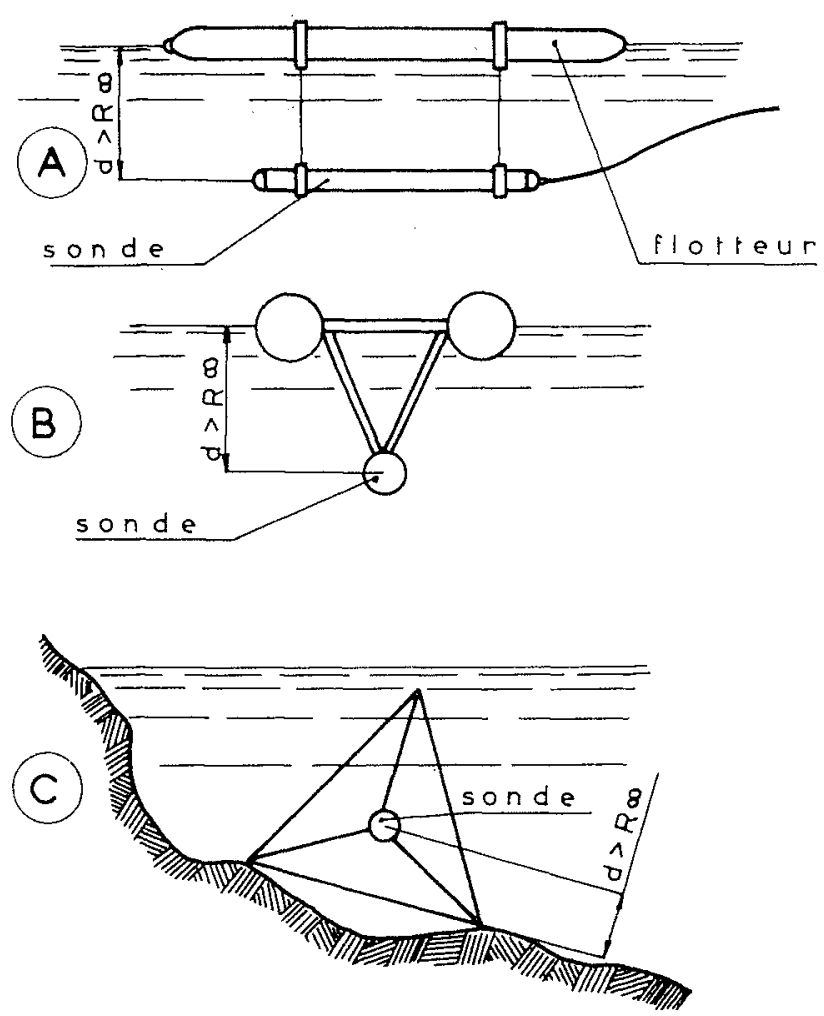

Fig. 3

Dispositifs d'immersion des sondes en rivière.

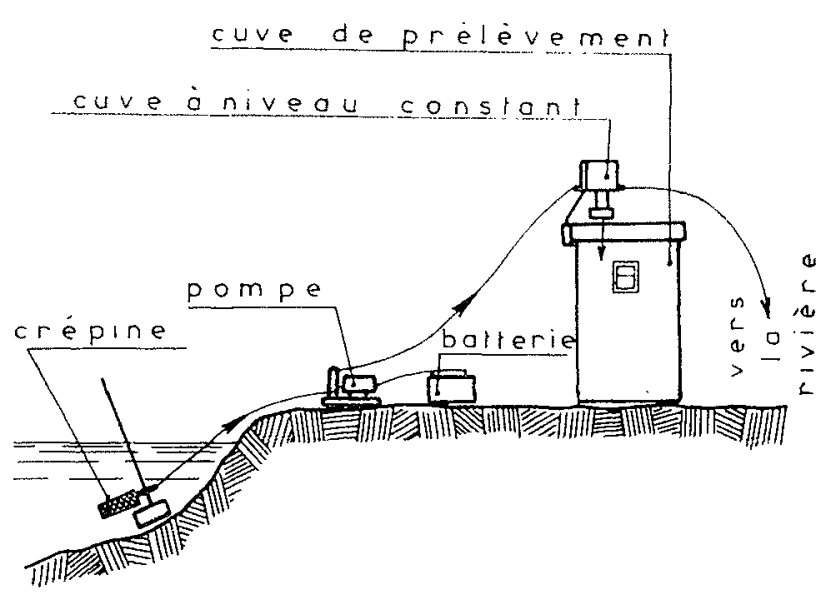

Dispositif de prélèvement d'un échantillon moyen.

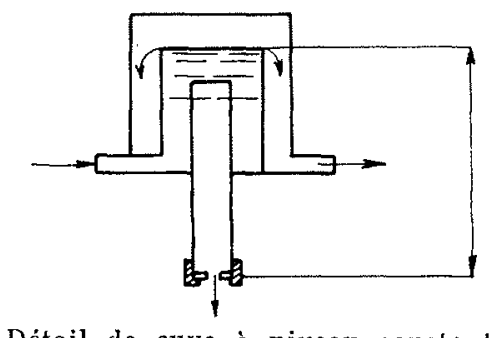

Détail de cuve à niveau constant.

FIG. 4

d'eau, ce qui est le cas des torrents de montagne.

Nous donnerons cependant plus loin les raisons qui nous font préférer une méthode de prélèvement à cette méthode de la sonde immergée.

\subsubsection{EChaNtillonNage continu.}

Certains expérimentateurs proposent de dériver une faible partie du courant de la rivière à travers une cuve dans laquelle plonge la sonde de détection. Ce procédé résout le problème de la géométrie d'étalonnage et libère des conditions de volume infini. Nous n'avons pas d'expérience de cette méthode, mais Iui préférons la suivante.

\subsubsection{Comptage D'Un ÉchantmLlon MOYEN.}

\subsubsection{Système de prélèvement:}

La figure 4 représente l'ensemble da système de prélèvement que nous utilisons pour les mesures en rivière. Ce système comprend une crépine et une pompe volumétrique entrainée par un moteur à courant continu alimenté par une batterie, qui assure un débit légèrement supérieur au débit $q$ désiré. Ce débit $q$ est déterminé par une cuve à niveau constant. Cette cuve est munie d'un ajustage calibré, dont l'interchangeabilité permet de choisir la valeur de $q$. Une partie de l'eau entrant dans la cuve à niveau constant est renvoyée à la rivière. 
Le débit de la pompe, voisin de $50 \mathrm{~cm}^{3} \mathrm{~s}^{-1}$, varie approximativement de $1 \%$ par heure; la hauteur du liquide dans la cuve à niveau constant est de $100 \mathrm{~mm}$; on est donc assuré que le débit de prélèvement est constant à mieux que $0,1 \%$.

Le taux de renouvellement de la solution dans cette cuve est de $1 / 3 \mathrm{~s}^{-1}$; on peut donc négliger "l'effet de capacité» dans le volume de cette cuve, étant donné les temps de passage des vagues d'activité.

\subsubsection{Mesure du débit de prélévement:}

La cuve de prélèvement est munie de deux fenêtres opposées, sur lesquelles est tracé un trait de repère définissant un volume de $18 \mathrm{l}$.

Le débit $q$ est déterminé en mesurant le temps nécessaire pour recueillir un volume donné, voisin de $18 \mathrm{l}$. On arrête le prélèrement avant que l'eau ait atteint le repère de 181 , car les cuves ne sont en général pas horizontales et plusieurs prélèvements sont effectués simultanément par un seul opérateur.

Le temps de prélèvement est mesuré. Puis, après avoir établi l'horizontalité de la cuve à l'aide d'un système tripode à vis calantes et un niveau, on ajuste le niveau an trait de repère en versant un volume connu d'eau. On en déduit le volume effectivement prélevé.

\subsubsection{Cuves et sondes de comptage:}

La cuve qui a servi au prélèvement de l'échantillon est également utilisée pour son comptage ainsi que pour le comptage de la prise aliquote. La sonde de détection est immergée dans la cuve, maintenue dans une géométrie reproductible à l'aide d'un système approprié. Son cristal se trouve au milieu du volume d'eau, dont le diamètre est de $30 \mathrm{~cm}$.

La plupart de nos essais ont été effectués avec une sonde constituée d'un compteur à haute efficacité LCT du type 13 G 14, dont la cathode a une surface de $180 \mathrm{~cm}^{2}$.

Nous utilisons de plus en plus une sonde à scintillations avec un cristal en INa (T1) de $1^{\prime \prime} \times 1^{\prime \prime} 1 / 2$, dont la stabilité est très satisfaisante à condition de prendre des précautions pour la maintenir à température constante. Le seuil de discrimination est voisin de $30 \mathrm{keV}$.

Les caractéristiques de ces sondes avec les cuves de 181 et pour ${ }^{82} \mathrm{Br}$ sont :

Sonde 13 G 14 :

- bruit de fond......

$725 \mathrm{cpm}$

-.. sensibilité. . . .....

$150 \mathrm{cpm} / \mu . \mathrm{c} / \mathrm{m}^{3}$

Sonde à scintillations:

- bruit de fond..... $4620 \mathrm{cpm}$

- sensibilité. ...... $1160 \mathrm{cpm} / \mu \mathrm{c} / \mathrm{m}^{3}$

Des essais nous ont montré qu'un facteur 10 peut être gagné sur la sensibilité de la sonde à scintillation en concentrant l'activité contenuc dans les 18 I d'eau sur une résine que l'on dispose contre le cristal pour la mesure.

\subsubsection{Activité à injecter :}

L'activité à injecter est fonction du temps de passage $T$ de la vague d'activité, du débit $Q$, qu'il faut évaluer approximativement, et de l'activité a qu'il est nécessaire de prélever pour obtenir la précision statistique désirée.

Pour un temps de comptage de $30 \mathrm{mn}$ de l'échantillon, de $15 \mathrm{mn}$ pour le bruit de fond, et un écart type relatif de $0,2 \%$, cette activité est $a=0,23 \mu c$ de ${ }^{82} \mathrm{Br}\left(13 \mu c / \mathrm{m}^{3}\right)$ avec notre sonde à scintillation.

L'activité à injecter est :

$$
\mathrm{A}=a \frac{q}{\mathrm{Q}}
$$

$q$ étant déterminé par $\mathrm{T}$.

L'évaluation de T n'est pas très difficile dans le cas des petites rivières et des conduites forcées.

La relation donnée plus haut pour les grands canaux donne des résultats satisfaisants, mais de sérieuses difficultés demeurent pour les fleuves.

Ces considérations et les essais préliminaires à la fluorescéine nous ont conduit à utiliser des activités de l'ordre de quelques dizaines de millicuries par mètre cube par seconde dans les jaugeages de torrents alpestres dont les débits étaient inférieurs à $10 \mathrm{~m}^{3} / \mathrm{s}$.

\subsection{Avantages de la méthode de l'échantillon moyen.}

La méthode d'intégration que nous venons d'exposer présente sur les deux autres procédés classiques d'intégration des avantages sur certains points importants que nous rappelons ou examinons ci-dessous :

$1^{\circ}$ On évite avec cette méthode de placer une sonde en "milieu infini» dans le courant à mesurer, opération difficile ou impossible dans les rivières à faibles tirants d'eau.

$2^{\circ}$ Elle rend possible la prise d'un échantillon moyen au centre et sur les bords de l'écoulement afin de vérifier que la condition de bon mélange est satisfaite. II ne serait pas possible d'immerger une sonde en milieu infini près des bords d'une rivière.

$3^{\circ}$ Dans le comptage d'un échantillon moyen, on peut se fixer a priori l'erreur statistique.

$4^{\circ}$ Le comptage d'un échantillon moyen permet éventuellement d'effectuer des corrections de perte de comptage. Ces corrections sont possibles 
avec une sonde immergée au prix d'un enregistrement des variations de la fréquence de comptage.

$5^{\circ}$ L'activité à injecter est assez critique avec une sonde immergée à compteurs GM, comme le montrent les courbes de la figure 5, qui représentent, pour différents types de détecteurs, les variations de l'erreur statistique et de l'erreur due aux pertes de comptage.

$6^{\circ}$ Il est avantageux d'effectuer le comptage de l'échantillon et de l'activité de référence à des dates voisines, étant donné les changements de gain de l'ensemble électronique, les corrections de décroissance d'activité, et éventuellement la décroissance d'activités parasites.

$7^{\circ}$ La méthode de prélèvement autorise 1'utilisation d'une. sonde à scintillation.

$8^{\circ}$ Cette méthode permet de concentrer l'activité afin d'accroître l'efficacité de comptage. C'est de plus la seule qui soit utilisable avec le tritium.

$9^{\circ}$ Les points $3^{\circ}, 7^{\circ}$ et $8^{\circ}$ conduisent à une réduction au minimum de l'activité injectée.

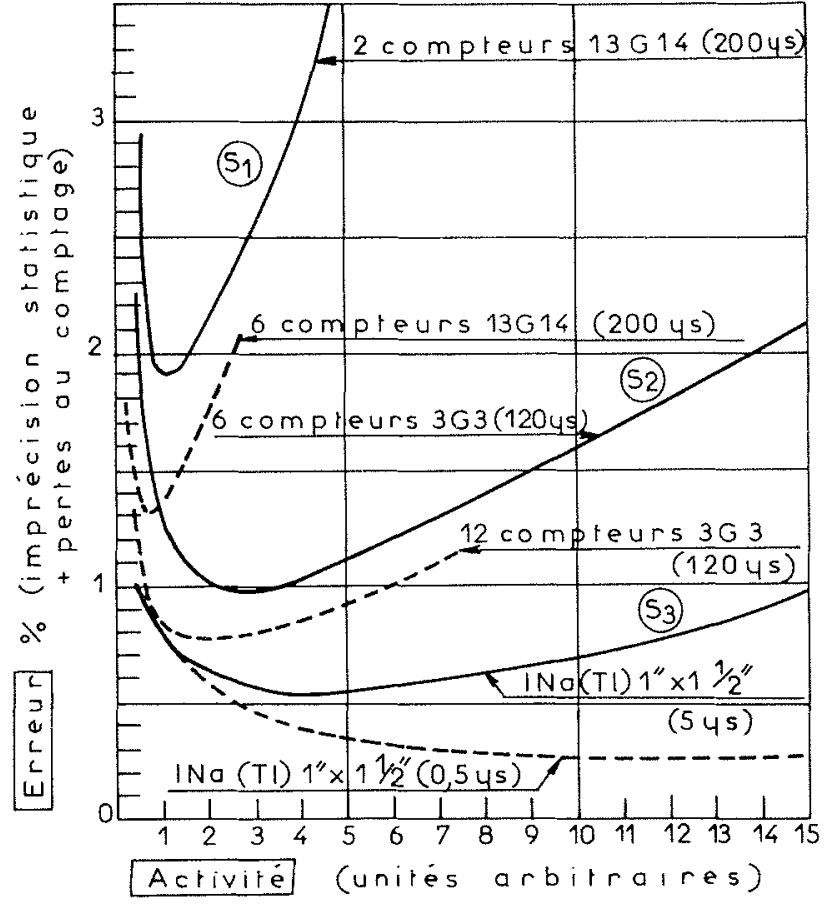

Frg. 5

Erreur de mesure en fonction de l'activité, pour diff'érents types de sondes.

\section{5. - QUESTION DE SECURITE}

Un règlement régit en France l'application des radioéléments dans le domaine de l'hydrologie. Sont autorisées les applications de traceurs de période inférieure à 8 jours, à condition de montrer qu'en aucun point de l'écoulement où l'on puisse capter de l'eau pour la boisson ou l'arrosage, la concentration du traceur dépasse la concentration maximale admissible.

Ce règlement, qui pourrait paraître exagérément dur, nous semble l'expression de la sagesse.
Une autorisation soumise à un examen spécial est nécessaire pour les traceurs dont la période excède 8 jours.

Cette clause, qui ne vise pas à éliminer des traceurs comme le tritium, met l'accent sur l'impératif d'un examen strict des conséquences de l'introduction, dans un milieu naturel d'éléments à longues périodes, tant du point de vue de la sécurité que de la contamination d'expériences ultérieures.

\section{6. - RESULTATS EXPERIMENTAUX}

Nous avons réalisé sept campagnes de mesures de débits de rivières et 3 campagnes de mesures de débits en conduites forcées; à titre d'exemple, nous donnerons les résultats d'une campagne de chaque type.

Le brome 82 a été adopté pour ces essais, car ce traceur a la réputation d'être peu adsorbé sur les terrains, comme l'ont montré certaines études sur la sélection de traceurs pour l'hydrologie souterraine. De plus, le brome 82 a l'une des concentrations maximales admissibles les plus élevées.

Nous pouvons distinguer deux périodes dans nos essais. Dans la première, la prise de partie aliquote du traceur se faisait sur le terrain; dans la seconde, nous utilisions au laboratoire une cellule spécialisée pour cette opération. Dans le deuxième cas, nous avons rencontré des diffi- 
Tableau I

Résullats des essais effectués à Chatou du 18 au 23 juin 1963 sur une condluite d'eau expérimentale

\begin{tabular}{|c|c|c|c|c|c|c|}
\hline $\begin{array}{c}N^{\circ} \\
\text { DE LA } \\
\text { SECTION }\end{array}$ & $\begin{array}{c}\text { POSITION } \\
\text { DU } \\
\text { RRÉLĖVEMENT }\end{array}$ & $\begin{array}{c}\text { DébTt } \\
\text { MESURÉ } \\
\text { (lis }\end{array}$ & $\begin{array}{c}\text { DÉBIT } \\
\text { VRAI } \\
(1 / \mathrm{s}) \\
\end{array}$ & $\begin{array}{r}\varepsilon_{1} \\
(\%) \\
\end{array}$ & $\begin{array}{r}\varepsilon_{2} \\
(\%) \\
\end{array}$ & $\begin{array}{r}\varepsilon_{3} \\
(\%) \\
\end{array}$ \\
\hline 1 & $\begin{array}{c}\text { Section } 1 \text { Centre } \\
-\quad 1 \text { Paroi } \\
-\quad 5 \text { Centre } \\
-\quad 5 \text { Paroi }\end{array}$ & $\begin{array}{l}54,5 \\
58,6 \\
53,7 \\
56,8\end{array}$ & 56,3 & $\begin{array}{l}-\quad 3,2 \\
+\quad 4,1 \\
+\quad 4,6 \\
+\quad 0,9\end{array}$ & -1 & $\begin{array}{l}7,3 \\
4,2\end{array}$ \\
\hline 2 & $\begin{array}{c}\text { Section } 1 \text { Centre } \\
-\quad 1 \text { Paroi } \\
-\quad 5 \text { Centre } \\
-\quad 5 \text { Paroi }\end{array}$ & $\begin{array}{l}144,5 \\
144,5 \\
149,8 \\
144\end{array}$ & 147,9 & $\begin{array}{l}-\quad 1,6 \\
-\quad 1,6 \\
-\quad 1,3 \\
-\quad 2,6\end{array}$ & $-1,5$ & $\begin{array}{l}0 \\
3,8\end{array}$ \\
\hline 3 & $\begin{array}{c}\text { Section } 2 \text { Centre } \\
=\quad 2 \text { Paroi } \\
=\quad 5 \text { Centre } \\
-\quad 5 \text { Paroi }\end{array}$ & $\begin{array}{r}119,9 \\
91,2 \\
100,9 \\
96,2\end{array}$ & 147,9 & $\begin{array}{l}-19 \\
-38 \\
-32 \\
-35\end{array}$ & -31 & $\begin{array}{r}28,7 \\
4,7\end{array}$ \\
\hline 4 & $\begin{array}{c}\text { Section } 2 \text { Centre } \\
-\quad 2 \text { Paroi } \\
=\quad 5 \text { Centre } \\
-\quad 5 \text { Paroi }\end{array}$ & $\begin{array}{l}77 \\
77,1 \\
76 \\
76,3\end{array}$ & 75,6 & $\begin{array}{l}+\quad 1,8 \\
+\quad 2,0 \\
+\quad 0,5 \\
+\quad 0,9\end{array}$ & $+1,3$ & $\begin{array}{l}0,13 \\
0,4\end{array}$ \\
\hline 5 & $\begin{aligned} & \text { Section } 3 \text { Centre } \\
&-\quad 3 \text { Paroi } \\
&-\quad 4 \text { Centre } \\
&-\quad 4 \text { Paroi }\end{aligned}$ & $\begin{array}{l}167,5 \\
168,5 \\
153,3 \\
174\end{array}$ & 181,4 & $\begin{array}{l}7,7 \\
-\quad 7,2 \\
-15,5 \\
-\quad 4,1\end{array}$ & $-8,5$ & $\begin{array}{r}0,6 \\
12,5\end{array}$ \\
\hline 6 & $\begin{array}{c}\text { Section } 1 \text { Centre } \\
-\quad 1 \text { Paroi } \\
-\quad 4 \text { Centre } \\
-\quad 4 \text { Paroi }\end{array}$ & $\begin{array}{l}134,7 \\
140,8 \\
134,1 \\
133,7\end{array}$ & 134,77 & $\begin{array}{ll} & 0 \\
+\quad 4,5 \\
-\quad 0,5 \\
-\quad 0,7\end{array}$ & $+\quad 0,75$ & $\begin{array}{l}4,4 \\
0,3\end{array}$ \\
\hline 7 & $\begin{array}{c}\text { Section } 3 \text { Centre } \\
-\quad 3 \text { Paroi } \\
-\quad 4 \text { Centre } \\
-\quad 4 \text { Paroi }\end{array}$ & $\begin{array}{l}125,7 \\
124 \\
126 \\
126,9\end{array}$ & 136,9 & $\begin{array}{l}-\quad 8,2 \\
-\quad 9,4 \\
-\quad 8 \\
-\quad 7,3\end{array}$ & $-8,2$ & $\begin{array}{l}1,3 \\
0,7\end{array}$ \\
\hline 8 & $\begin{array}{c}\text { Section } 3 \text { Centre } \\
=\quad 3 \text { Paroi } \\
-\quad 4 \text { Centre } \\
-\quad 4 \text { Paroi }\end{array}$ & $\begin{array}{l}137,2 \\
136,9 \\
138,2 \\
134,6\end{array}$ & 137 & $\begin{array}{ll}+ & 0,2 \\
- & 0,1 \\
+ & 0,7 \\
- & 1,7\end{array}$ & $-0,2$ & $\begin{array}{l}0,2 \\
2,5\end{array}$ \\
\hline 9 & $\begin{array}{l}\text { Section } 2 \text { Centre } \\
-\quad 2 \text { Paroi } \\
-\quad 5 \text { Centre } \\
-\quad 5 \text { Paroi }\end{array}$ & $\begin{array}{l}147,6 \\
146,4 \\
148,7 \\
148\end{array}$ & 148,7 & $\begin{array}{l}-\quad 0, \pi \\
-\quad 1,5 \\
-\quad 0 \\
-\quad 0,5\end{array}$ & $-0,7$ & $\begin{array}{l}0,8 \\
0,5\end{array}$ \\
\hline 10 & $\begin{array}{c}\text { Section } 2 \text { Centre } \\
-\quad 2 \text { Paroi } \\
-\quad 5 \text { Centre } \\
-\quad 5 \text { Paroi }\end{array}$ & $\begin{array}{l}148,8 \\
148,3 \\
148,6 \\
147,5\end{array}$ & 148,7 & $\begin{array}{l}+\quad 0,1 \\
-\quad 0,3 \\
-\quad 0,1 \\
-\quad 0,8\end{array}$ & $-0,3$ & $\begin{array}{l}0,3 \\
0,7\end{array}$ \\
\hline 11 & $\begin{array}{c}\text { Section } 3 \text { Centre } \\
-\quad 3 \text { Paroi } \\
=\quad 5 \text { Centre } \\
-\quad 5 \text { Paroi }\end{array}$ & $\begin{array}{l}143,1 \\
142 \\
140,4 \\
140,4\end{array}$ & 148,6 & $\begin{array}{l}-\quad 3,7 \\
-\quad 4,4 \\
-\quad 5,5 \\
-\quad 5,5\end{array}$ & $-4,9$ & $\begin{array}{l}0,7 \\
0\end{array}$ \\
\hline
\end{tabular}


cultés dues à des produits de décomposition de la molécule $\mathrm{BrNH}_{4}$ au cours de l'irradiation; ces produits contaminaient les prises aliquotes.

Pour les mesures de débits en conduites forcées d'usines hydroélectriques, les valeurs de référence étaient fournies par des moulinets, tandis qu'en rivière nous utilisions les résultats de mesures de dilution de bichromate de sodium. La précision de cette dernière méthode est de l'ordre de 2 à $3 \%$.

Les distances entre les points d'injection et de mesures, dans le cas des rivières de débit inférieur à $10 \mathrm{~m}^{3} / \mathrm{s}$, étaient comprises entre 200 et $600 \mathrm{~m}$, et les temps de prélèvement ne dépassaient pas $30 \mathrm{mn}$.

\subsection{Mesures sur une conduite expérimentale. Chatou 18-23 juin 1962.}

Le tableau I résume les mesures effectuées sur une conduite expérimentale de $30 \mathrm{~cm}$ de diamètre et dont le débit, déterminé à l'aide d'une bâche de pesée, était connu avec une précision de $0,2 \%$.

Cinq sections de prélèvements étaient aménagées, dans la conduite, comportant chacune deux tubes, l'un prélevant au centre de la section, l'autre contre la paroi. Les distances de ces sections à la section d'injection étaient :

- pour la section 1: $16 \mathrm{~m}$;

- pour la section 2: $22 \mathrm{~m}$;

- pour la section 3:28 m;

- pour la section 4: $35 \mathrm{~m}$;

- pour la section 5: $43 \mathrm{~m}$.

A chaque injection, 4 prẻlèvements étaient réalisés avec un débit de $0,04 \mathrm{l} / \mathrm{s}$. Les temps de prélèvements variaient de 120 à $300 \mathrm{~s}$.

Pour les injections 1 à 6 , le débit de trop-plein des bacs à niveau constant était trop faible et a pu même s'annuler. Les prélèvements des mesures 7 et 8 ont été faits sans cuve à niveau constant.

Les valeurs $\varepsilon_{1}, \varepsilon_{2}$ et $\varepsilon_{3}$ portées dans le tableau sont définies comme suit :

$\varepsilon_{1}=100 x \frac{\text { débit mesuré - débit vrai }}{\text { débit vrai }}$

pour chaque mesure;

$\varepsilon_{2}=\frac{\Sigma \varepsilon_{1}}{4}$ pour chaque injection;

$\varepsilon_{3}=$ écarts relatifs entre les deux débits mesurés dans une section. Cette valeur représente les variations de l'expression $\int_{\mathrm{T}} c d t$.

On peut attribuer à une prise défectueuse d'aliquote les divergences représentées par $\varepsilon_{*}$ pour les injections $2,3,5,7$ et 11 . Les divergen- ces sur $\varepsilon_{3}$, c'est-à-dire sur $\int_{\mathrm{T}} c d t$, aux injections $1,3,5,6$ et 8 , peuvent être attribuées aux variations du débit de prélèvement.

Il est important de remarquer cependant que : sur 44 valeurs de $\varepsilon_{1}, 16$ sont inférieures à $1 \%$; sur 11 valeurs de $\varepsilon_{2}, 5$ sont inférieures à $1 \%$; sur 22 valeurs de $\varepsilon_{3}, 13$ sont inférieures à $1 \%$.

On peut espérer, à la suite de ces essais et, de jaugeages que nous avons effectués ou de conduites forcées, qu'il sera possible d'obtenir pour ce type de mesure une précision voisine, sinon meilleure, de $1 \%$ par la méthode d'intégration radioactive.

\subsection{Mesures de débit de rivières. Roselend 26-28 septembre 1962.}

Au cours des essais précédents en rivière, on a pu mettre en évidence une divergence au plus de 3 à $4 \%$, entre nos résultats et cenx de la méthode de dilution de bichromate.

Le tableau II donne les résultats des essais, effectués à Roselend en septembre 1962, au cours desquels 5 injections ont été faites; quatre prélèvements dans une même section de la rivière correspondaient à chaque injection.

Les débits mesurés sont erronés de $20 \%$ environ, car les ampoules des prises aliquotes itaient contaminées.

Un examen de la dispersion entre les quatre résultats d'une mếme mesure apporte une excellente vérification de la relation $\int_{\mathrm{T}} c d t=$ Cte.

Les valeurs de la dernière colonne qui représentent les écarts de chaque mesure par rapport à la valeur moyenne excèdent rarement $1 \%$, excepté dans les cas suivants :

a) Injection $n^{*} 1$; prélèvements 1 et 4 . Les écarts de $+2,8 \%$ et $-4 \%$ montrent que la distance d'injection est trop faible. Cette dislance, après avoir été portée de 300 à $400 \mathrm{~m}$, s'est montrée suffisante pour donner un bon mélange.

b) Injection $\mathrm{n}^{\circ} 3$; prélèvement 4 . Nous n'avons pas trouvé de causes à cet écart.

c) Injection $n^{\circ} 5$; prélèvement 2 . La valeur de $2,9 \%$ peut s'expliquer par une perte d'activité au prèlèvement. En effet, ce dernier a été réalisé dans un courant secondaire à faible renouvellement. Il est très probable que la valeur d'activité en ce point a été plus longue que le temps de prélèvement.

Nous pouvons dégager de cet essai, et de nos expériences précédentes, une première conclusion. Etant donné, d'une part la précision de la méthode de référence et par ailleurs, l'amélioration prévue du procédé de prise aliquote, on peut situer sans être optimiste entre 2 et $4 \%$ la précision des jaugeages de torrents alpestres par la méthode d'intégration radioactive. 
Tablene II

Résultats des essais de mesure de débits de rivière effectués à Roselend da 26 au 28 septembre 1962

\begin{tabular}{|c|c|c|c|c|c|}
\hline DE L'INJECTION & $\begin{array}{l}\text { DATE ET HEURE } \\
\text { DE L'INJECTION }\end{array}$ & $\begin{array}{c}\text { N" } \\
\text { DU PRÉLĊVEMENT }\end{array}$ & $\begin{array}{l}\text { DÉBIT MEsURÉ } \\
\qquad \mathrm{Qi} \mathrm{m}^{3} / \mathrm{s}\end{array}$ & $\begin{array}{c}Q_{m}=\frac{Q_{b}}{4} \\
\left(\mathrm{~m}^{3} / \mathrm{s}\right)\end{array}$ & $\begin{array}{c}100 \times \frac{Q_{m}-Q_{\varepsilon}}{Q_{m}} \\
(\%)\end{array}$ \\
\hline 1 & $26 / 9-16$ l 30 & $\begin{array}{l}1 \\
2 \\
3 \\
4\end{array}$ & $\begin{array}{l}3,68 \\
3,78 \\
3,75 \\
3,93\end{array}$ & 3,785 & $\begin{array}{l}+2,8 \\
+0,1 \\
+0,9 \\
-4\end{array}$ \\
\hline 2 & $27 / 9-9 \mathrm{~h} 45$ & $\begin{array}{l}1 \\
2 \\
3 \\
4\end{array}$ & $\begin{array}{l}3,725 \\
3,70 \\
3,695 \\
3,70\end{array}$ & 3,705 & $\begin{array}{l}-0,5 \\
+0,1 \\
+0,3 \\
+0,1\end{array}$ \\
\hline 3 & $27 / 9-16$ Һ 50 & $\begin{array}{l}1 \\
2 \\
3 \\
4\end{array}$ & $\begin{array}{l}3,556 \\
3,543 \\
3,547 \\
3,438\end{array}$ & 3,521 & $\begin{array}{l}-1 \\
-0,6 \\
-0,7 \\
+2,3\end{array}$ \\
\hline 4 & $28 / 9-9$ h 55 & $\begin{array}{l}1 \\
2 \\
3 \\
4\end{array}$ & $\begin{array}{l}3,275 \\
3,267 \\
3,277 \\
3,237\end{array}$ & 3,264 & $\begin{array}{r}-0,3 \\
-0,1 \\
-0,4 \\
+0,8\end{array}$ \\
\hline 5 & $28 / 9-16$ l 30 & $\begin{array}{l}1 \\
2 \\
3 \\
4\end{array}$ & $\begin{array}{l}3,781 \\
3,961 \\
3,804 \\
3,810\end{array}$ & & $\begin{array}{l}+1,3 \\
-2,9 \\
+0,9 \\
+0,7\end{array}$ \\
\hline
\end{tabular}

\section{CONCLUSION}

Nous avons montré qu'un pas important a été fait, à l'aide de la méthode d'intégration radioactive, vers les mesures de débits de grands cananx et fleuves pour lesquelles le tritium semble le plus favorisé. Si, pour la mesure des débits en conduites forcées et de rivières, d'autres mé- thodes sont en compétition, complémentaires ou préférables, on dispose maintenant d'un nouveau procédé qui ne demande, pour ètre bien assis, qu'un complément d'étude sur le phénomène d'adsorption du traceur et le verdict de centaines d'essais.

\section{BIBLIOGRAPHIE}

(1) Hull D. E. et Macomber M. - Proceeding of the Second International Conference on the Paceful Uses of Atomic Energy, Geneva, vol. 19, 324, United Nations, N.Y. (1958).

(2) Pérez R. - Contribution à l'étude en canal de la diffusion saline en régime permanent. Thèse présentée à l'Université de Toulouse le 26 mars 1963.

(3) Rimiar G. M. - Trudy GGI, fascicule 36, 1960. 


\section{DIS C US SION}

Président : M. BECKER

M. le Président remercie M. Pérez au nom du Comité Technique, d'avoir bien voulu exposer cette méthode, et ouvre la discussion.

M. Raud demande si, lorsque l'on emploie dans les cours d'eau des méthodes de jaugeage chimique, les teneurs des eaux en limons constituent une difficulté importante, surtout quand ces teneurs. sont très élevées comme cela arrive parfois dans certains pays. Il demande dans quelle mesure l'utilisation des traceurs radio-actifs permet de se libérer de ces sujétions.

M. PÉrez répond qu'il n'a pas fait d'expériences en rivières très chargées en limons, mais que, personnelnellement et a priori, il ne pense pas qu'il puisse y avoir incidence.

Sur la remarque de M. Raud concernant l'importance des absorptions de bichromate de sodium par les limons, M. PÉREZ répond qu'en effet les limons réduisent parfois le bichromate à l'état de $\mathrm{Cr}_{3}$, à partir duquel on ne peut plus faire d'analyse par les méthodes classiques. Ceci peut être réparé par une réoxydation des échantillons.

M. Breusse met alors l'accent sur la difficulté et le danger de manutention des parties aliquotes.

M. PÉREZ reconnaît la nécessité d'employer, pour cette méthode, des agents ayant l'habitude de manipuler des produits radio-actifs.

M. RÉméniéras remarque que les méthodes étudiées rentrent dans le cadre des méthodes de jaugeage dites " par dilution », en cours de normalisation par les soins de l'ISO (Sous-Comité TC $30 / \mathrm{SC} 1$ ). Alors que, jusqu'à ces dernières années, on injectait dans l'écoulement à jauger un débit (ou une quantité) connu de produit chimique (chlorure de sodium, bichromate de sodium, etc...), on a essayé plus récemment d'injecter un débit (ou une quantité) connu de produit radioactif. Dans les deux cas, le principe de la méthode reste le même et la condition de bon mélange est aussi impérative.

Les essais en cours de divers côtés devront comparer, d'une part la précision susceptible d'être obtenue (dans la mesure des concentrations relatives notamment) et, d'autre part, le prix et la plus ou moins grande facilité d'emploi de chácun des deux types de "marqueurs》 visés. Il semble difficile de délimiter dès maintenant le champ d'application pratique des produits radioactifs dans le domaine des méthodes de dilution; il semble pourtant que l'emploi du tritium pourrait permettre, après mise au point, le jaugeage de très gros débits (tels que ceux du Fleuve Jaune par exemple).

M. le Président remercie encore M. Pérez d'avoir su, d'un très important travail, dégager les éléments essentiels et les présenter de façon très claire.

M. le Président espère que cette conférence aura onvert des horizons qui appelleront des applications probablement fécondes.

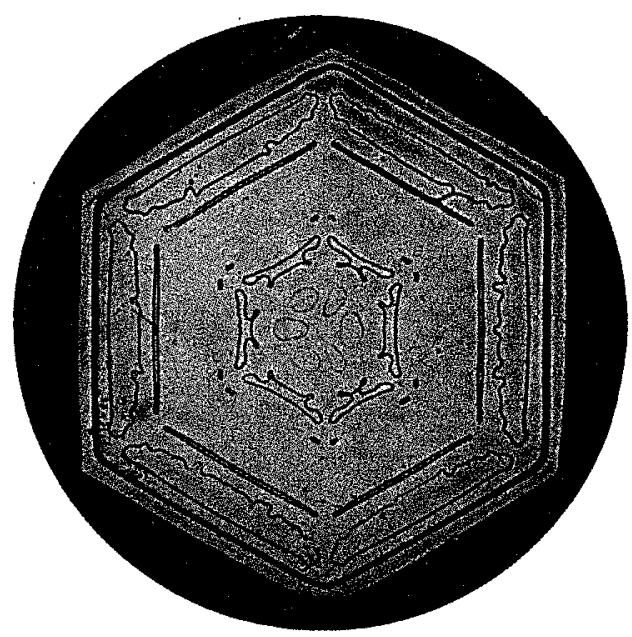

